\title{
Manifestaciones cutáneas en pacientes con síndrome de Down
}

Skin manifestations in Down's syndrome

\author{
Catalina Restrepo', Luis Alfonso Correa², Margarita María Velásquez ${ }^{3}$ \\ 1. Médica, residente de tercer año de Dermatología, Sección de Dermatología, Facultad de Medicina, Universidad de Antioquia, Medellín, Colombia \\ 2. Médico patólogo; profesor de Dermatopatología, Centro de Investigaciones Dermatológicas, CIDERM; Grupo de Investigaciones Dermatológicas, \\ GRID, Sección de Dermatología, Facultad de Medicina, Universidad de Antioquia, Medellín, Colombia \\ 3. Médica dermatóloga, doctora en Ciencias Básicas Biomédicas con énfasis en Inmunología; Centro de Investigaciones Dermatológicas, CIDERM, \\ Sección de Dermatología, Facultad de Medicina, Universidad de Antioquia, Medellín, Colombia
}

\section{Resumen}

El síndrome de Down es una enfermedad congénita asociada a retraso mental y características físicas distintivas. Se presenta con diversos signos y síntomas en piel, muchos de los cuales pueden explicarse por la xerosis; otros son propios de la edad del paciente y se ven agravados por las características genéticas de la cromosomopatía o por las enfermedades asociadas. Se pueden presentar siringomas, alopecia areata, elastosis perforans serpiginosa y calcinosis cutis milioide (milia-like), entre otras. Es frecuente la presencia de infecciones bacterianas y virales relacionadas con el déficit inmunológico.

PALABRAS CLAVE: síndrome de Down, trisomía 21, manifestaciones cutáneas, inmunodeficiencias.

\section{Summary}

Down syndrome is a congenital disorder associated with mental retardation and physical features. From a dermatological standpoint, these patients have a variety of signs and symptoms, many of which can be explained by xerosis, others are specific to the patient's age and compounded by the chromosomal genetic characteristics associated conditions. Patients with Down syndrome may have xerosis, syringoma, alopecia areata, perforating elastosis and milia-like calcinosis cutis. Often the presence of infections such as folliculitis, furunculosis, fungal infections and warts, among others, related to immune deficiency.

KEY WORDS: Down syndrome, 21 trisomy, skin manifestations, immune deficiencies

\section{Correspondencia:}

Margarita María Velásquez

Email:

mmvelasquez@yahoo.com

Recibido: 13 de agosto de 2012.

Aceptado: 19 de diciembre de 2012.

No se reportan conflictos de intereses.

\section{Introducción}

El síndrome de Down es la anormalidad cromosómica más común en los recién nacidos vivos y es la forma más frecuente de retraso mental. Es causada por una aberración en el cromosoma 21, resultando tres copias en lugar de dos por una falla en la meiosis del óvulo o, en algunos casos, del espermatozoide. Esto da como resultado un cariotipo de 47 cromosomas, a diferencia del normal de 46. Aparece con una frecuencia de 1 entre cada 700 a 800 recién nacidos, $95 \%$ tienen una trisomía simple, 3 a $4 \%$ se deben a translocación y, aproximadamente, el 1 a 2\% son mosaicos. El síndrome de Down se caracteriza por una variedad de rasgos dismórficos, malformaciones congénitas, otros problemas de salud y condiciones médicas, pero no todas las manifestaciones clínicas se presentan en los individuos afectados ${ }^{1}$. 
Los pacientes con síndrome de Down presentan un fenotipo característico, alteraciones sistémicas diversas y un aumento de la frecuencia de problemas dermatológicos. El fenotipo en la piel incluye pliegue palmar transverso y xerosis, macroglosia y lengua fisurada ${ }^{2}$. Entre las manifestaciones sistémicas se destacan las cardiopatías congénitas, las alteraciones oculares y tiroideas, la enfermedad celiaca, la carotinemia, el envejecimiento prematuro y la disfunción inmunológica que aumenta el riesgo de infecciones y de neoplasias hematológicas.

Debido a los avances médicos, la edad promedio de muerte se ha incrementado a 49 años y la esperanza de vida de un persona de un año de edad con síndrome de Down es, en la actualidad, de más de 60 años ${ }^{1}$. Por lo tanto, los dermatólogos tienen más probabilidades de encontrar un mayor espectro de los trastornos dermatológicos que se presentan en estos pacientes y de hallar nuevas publicaciones en la literatura científica.

Esta revisión se enfoca en las dermatosis que se presentan con mayor frecuencia en el síndrome de Down. Se describen primero las principales alteraciones de la respuesta inmunitaria, lo que contribuye a comprender mejor las manifestaciones dermatológicas.

\section{Alteraciones en el sistema inmunitario}

En el síndrome de Down se presenta una alta mortalidad debido a infecciones, principalmente neumonía, y, además, existe un riesgo alto de desarrollar neoplasias hematológicas malignas, especialmente leucemia ${ }^{3}$. Es el síndrome genético identificado que más frecuentemente se asocia a defectos inmunitarios ${ }^{4}$.

En la década de 1970 se propuso la hipótesis de que en el síndrome de Down debía haber más alteraciones inmunológicas, debido a la mayor frecuencia de neoplasias hematológicas, enfermedades autoinmunitarias e infecciones, y la frecuencia de portadores de antígeno de superficie de hepatitis $\mathrm{B}^{5}$. En la actualidad, los defectos inmunitarios en el síndrome de Down están parcialmente descritos y no completamente entendidos.

\section{Inmunidad innata}

Los estudios muestran una reducción de las células NK $\mathrm{CD}_{16}{ }^{+} \mathrm{CD} 56^{+}$en los niños, pero el número se restablece posteriormente en los adultos-. Lo que más se ha estudiado ha sido la función de las células, en la cual se ha observado una reducción de la capacidad quimiotáctica de los leucocitos polimorfonucleares y de los fagocitos mononucleares ${ }^{8}$. Los resultados sobre la capacidad fa- gocítica de los polimorfonucleares son variables; en unos estudios se aprecia disminución y en otros no hay variaciones con relación a los controles. Lo que se ha encontrado más constantemente es el aumento de la apoptosis de neutrófilos y eosinófilos, y la reducción de la capacidad citotóxica de las células $\mathrm{NK}^{5,8}$.

También se han sugerido trastornos en la secreción de citocinas, como IL-2, IL-7 e IL-10 9 , y deficiencia en las proteínas fijadoras de manosa7. Todos estos hallazgos contribuyen al aumento de la frecuencia de infecciones y de algunas manifestaciones cutáneas.

\section{Inmunidad adaptativa}

El timo en las personas con síndrome de Down presenta, desde edades tempranas, alteraciones de su desarrollo, con reducción del grosor de la corteza y de la zona córtico-medular, y depleción de los timocitos corticales $^{5}$. En los niños se ha observado disminución en las proporciones de timocitos $\mathrm{CD}_{1}^{+}, \mathrm{CD}_{3}^{+}, \mathrm{CD}_{4}^{+} \mathrm{y}$ $\mathrm{CD}^{+}{ }^{10}$. Además, muestra una reducción de los timocitos fenotípicamente maduros, los cuales tiene gran expresión del receptor de células T TCR $\alpha \beta$ y CD3 y la sobreexpresión del factor de necrosis tumoral $\alpha(\mathrm{FNT} \alpha)$ y del interferón (IFN)-g ${ }^{5,9}$. Esto indica una disfunción en los mecanismos de maduración del timo, con retraso en la maduración de linfocitos $\mathrm{T}$.

El número total de leucocitos y de linfocitos está disminuido en los niños con síndrome de Down de todas las edades ${ }^{11}$; esta reducción suele aminorarse conforme avanza la edad. En consecuencia, está reducido el número de células $\mathrm{T}$, sobre todo en los dos primeros años de vida y, por tanto, el número de células $\mathrm{T} \mathrm{CD}^{+}{ }^{+} \mathrm{CD}^{+}$; la proporción $\mathrm{CD}_{4}^{+} / \mathrm{CD}^{+}$es estable a lo largo de la vida, pero su valor en el síndrome de Down es menor que en la población control, lo que indica una mayor reducción del grupo de células $\mathrm{T} \mathrm{CD}_{4}^{+12}$.

Varios estudios han demostrado, también, disminución de linfocitos B como consecuencia de una disfunción en su expansión y maduración ${ }^{5}$. En cuanto a laproducción de inmunoglobulinas, se describe diversos defectos entre estos, hipogammaglobulinemia, hipergammaglobulinemia $\mathrm{G}$ y A después de los cinco años de edad, con altos o bajos niveles de IgG1 e IgG3 y bajos niveles de IgG2 e IgG4, con la disminución de los niveles de IgM en la adolescencia. Otros reportan disminución de la IgA en saliva y deficiencia de la producción de anticuerpos IgG específicos a las vacunas ${ }^{5,9}$.

\section{Envejecimiento de la piel}

El envejecimiento en el síndrome de Down se presenta 
de una manera acelerada. El inicio de los cambios depende de la edad y estos incluyen encanecimiento del pelo, pérdida del pelo, arrugas en la piel, menopausia, osteoporosis, osteoartritis, hipogonadismo, cambios inmunológicos y cataratas seniles ${ }^{13,14}$.

Existen teorías que podrían explicar esta aceleración. Una de ellas habla de una alteración en la reparación del ADN, con niveles basales significativamente más bajos de muchas enzimas de reparación del ADN que, además, se deterioran con el envejecimiento ${ }^{15}$. Otra posible explicación seria una alteración en el metabolismo de los radicales libres ${ }^{14}$. Los efectos de la exposición crónica al sol y el envejecimiento intrínseco de la piel, llevan a la generación de radicales libres. La dismutasa de superóxido, una enzima clave en metabolismo de los radicales libres, se encuentra en el cromosoma $21^{16}$. La sobreexpresión de la dismutasa de superóxido conduce al aumento de la producción de $\mathrm{H}_{2} \mathrm{O}_{2} \mathrm{y}$, por lo tanto, a una mayor producción de radicales citotóxicos y especies de oxígeno. Estos, a su vez, causan oxidación de lípidos de membrana celular, alterando la estructura y la función de los tejidos involucrados ${ }^{16}$.

En las TABLAS 1 Y 2 se describen las manifestaciones cutáneas en el síndrome de Down y su frecuencia.

\section{Dermatosis más frecuentes}

\section{Dermatitis atópica}

La prevalencia de dermatitis atópica en el síndrome de Down es contradictoria, pues en los estudios iniciales se encontraba una incidencia tan alta como $56,5 \%$ y una prevalencia de $50 \%{ }^{17,18}$. Posteriormente, con la introducción de los criterios diagnósticos de Hanifin y Rajka para la dermatitis atópica, esta prevalencia disminuyó hasta 3 a $4,9 \%{ }^{2,19}$.

En una revisión de la literatura científica más reciente, se encontró una prevalencia del $11 \%{ }^{20}$. La gran prevalencia de la dermatitis atópica en los estudios previos podría ser una sobreestimación ya que, sin criterios diagnósticos, signos aislados como la xerosis generalizada podrían ser mal interpretados como dermatitis atópica $^{21}$. La dermatitis atópica en estos pacientes puede ser grave y difícil de manejar, ya que a menudo se complica por presencia de liquen e impétigo causados por un aumento de la propensión a las infecciones.

\section{Dermatitis seborreica}

Su incidencia es mayor en comparación con la población normal. En los diferentes estudios se ha encontrado una concordancia en la incidencia, que está alrededor de
Dermatosis típicamente relacionadas

con el síndrome de Down

Elastosis perforans serpiginosa

Siringomas palpebrales o siringomas eruptivos

Calcinosis cutis idiopática de tipo miliar

Dermatosis papulosa folicular

Dermatosis más frecuentes

Xerosis

Dermatitis seborreica

Alopecia areata

Vitiligo

Queratosis palmo-plantar

Dermatitis atópica

Cutis marmorata

Queratosis pilar

Ictiosis

Acrocianosis

Queilitis

Foliculitis

Sarna noruega

Tinea corporis

Onicomicosis

Anetodermia

TABLA 1. Manifestaciones cutáneas en el síndrome de Down.

$30 \%{ }^{22}$. Algunos autores refieren que, posiblemente, la mayor prevalencia de foliculitis por Malassezia spp. en el síndrome de Down podría tener un papel patogénico en la dermatitis seborreica, pero aún no hay estudios que lo confirmen ${ }^{23}$.

\section{Alopecia areata}

Los niños con síndrome de Down presentan pelo más delgado, fino y, en ocasiones, hipopigmentado. La alopecia areata es una alopecia inflamatoria no cicatricial común, que se produce en 0,7 a 3,8 \% de los pacientes que acuden a la consulta de dermatología ${ }^{24}$.

En la literatura científica existe una asociación bien reconocida entre la alopecia areata y el síndrome de Down. Du Vivier y Munro, en 1967, fueron los primeros en hablar de esta asociación. Posteriormente, en 1976, 


\begin{tabular}{|c|c|c|c|c|c|c|}
\hline & $\begin{array}{c}\text { Cano MP. } \\
\qquad \begin{array}{c}(20) \\
n=90 \%\end{array}\end{array}$ & $\begin{array}{c}\text { Ferrando et al } \\
\text { (51) } \\
n=416 \%\end{array}$ & $\begin{array}{c}\text { Schepis C et al } \\
\begin{array}{c}\text { (2) } \\
n=203 \%\end{array}\end{array}$ & $\begin{array}{c}\text { Ercis } M \text { et al } \\
\qquad \begin{array}{c}(22) \\
n=71 \%\end{array}\end{array}$ & $\begin{array}{l}\text { Polenghi MM et al } \\
\begin{array}{c}\text { (52) } \\
n=96 \%\end{array}\end{array}$ & $\begin{array}{l}\text { Martin D } \\
\qquad \begin{array}{c}(53) \\
n=213 \%\end{array}\end{array}$ \\
\hline Dermatitis atópica & 11 & $63,4^{\star \star}$ & 4,9 & 1,4 & 8 & 56,5 \\
\hline Dermatitis seborreica & 18,8 & 21,6 & 8 & 30,9 & $\mathrm{NE}$ & 36 \\
\hline Alopecia areata & $\mathrm{NE}$ & 14 & 2,9 & 1,4 & 20 & 8,9 \\
\hline Vitiligo & $\mathrm{NE}$ & $\mathrm{NE}^{\star}$ & $\mathrm{NE}$ & NE & $\mathrm{NE}$ & 1,9 \\
\hline $\begin{array}{l}\text { Calcinosis cutánea } \\
\text { de tipo miliar }\end{array}$ & $\mathrm{NE}$ & $\mathrm{NE}$ & 2,9 & $\mathrm{NE}$ & $\mathrm{NE}$ & $\mathrm{NE}$ \\
\hline Siringomas & $\mathrm{NE}$ & $\mathrm{NE}$ & 12,3 & $\mathrm{NE}$ & $\mathrm{NE}$ & 39,2 \\
\hline $\begin{array}{l}\text { Elastosis perforans } \\
\text { serpiginosa }\end{array}$ & NE & $\mathrm{NE}$ & 0,49 & $\mathrm{NE}$ & $\mathrm{NE}$ & $\mathrm{NE}$ \\
\hline Onicomicosis & $\mathrm{NE}$ & $5^{\star \star \star}$ & 4,4 & $\mathrm{NE}$ & 8 & 67,8 \\
\hline Tinea corporis & NE & $5^{\star \star \star}$ & 1,9 & $\mathrm{NE}$ & NE & $\mathrm{NE}$ \\
\hline Anetodermia & $\mathrm{NE}$ & $\mathrm{NE}$ & 3,9 & $\mathrm{NE}$ & $\mathrm{NE}$ & $\mathrm{NE}$ \\
\hline Foliculitis & $\mathrm{NE}$ & 19,7 & 21,1 & $\mathrm{NE}$ & 26 & 10,3 \\
\hline Queilitis & $\mathrm{NE}$ & 11,3 & 2,4 & 5,6 & $\mathrm{NE}$ & $\mathrm{NE}$ \\
\hline Queratosis pilaris & 33,3 & $\mathrm{NE}$ & 2,3 & 2,8 & $\mathrm{NE}$ & 3,2 \\
\hline Psoriasis & $\mathrm{NE}$ & 5,2 & 1,4 & $\mathrm{NE}$ & 8 & 0,5 \\
\hline $\begin{array}{c}\text { Cutis marmorata/ livedo } \\
\text { reticularis }\end{array}$ & 44,4 & NE & 8,8 & 12,6 & $\mathrm{NE}$ & 8,4 \\
\hline Xerosis & 67,7 & $63,4^{\star}$ & 9,8 & $\mathrm{NE}$ & NE & 85 \\
\hline $\begin{array}{l}\text { Hiperqueratosis palmo- } \\
\text { plantar }\end{array}$ & 47,7 & $\mathrm{NE}$ & $\mathrm{NE}$ & 40,8 & $\mathrm{NE}$ & NE \\
\hline Acné & $\mathrm{NE}$ & 8,4 & $\mathrm{NE}$ & $\mathrm{NE}$ & $\mathrm{NE}$ & NE \\
\hline Lengua fisurada & $\mathrm{NE}$ & 72,1 & $\mathrm{NE}$ & $\mathrm{NE}$ & $\mathrm{NE}$ & $\mathrm{NE}$ \\
\hline Dermatitis del pañal & 22,2 & $\mathrm{NE}$ & $\mathrm{NE}$ & $\mathrm{NE}$ & $\mathrm{NE}$ & $\mathrm{NE}$ \\
\hline Manchas café con leche & 26,6 & NE & $\mathrm{NE}$ & $\mathrm{NE}$ & NE & $\mathrm{NE}$ \\
\hline Mancha mongólica & 62,2 & $\mathrm{NE}$ & $\mathrm{NE}$ & $\mathrm{NE}$ & $\mathrm{NE}$ & $\mathrm{NE}$ \\
\hline Eritema malar & 65,5 & NE & $\mathrm{NE}$ & $\mathrm{NE}$ & NE & $\mathrm{NE}$ \\
\hline
\end{tabular}

TABLA 2. Frecuencia de las manifestaciones cutáneas en el síndrome de Down

$\mathrm{N}$ : número de pacientes del estudio; ${ }^{\star} \mathrm{NE}$ : no evaluado; ${ }^{\star \star}$ : no hacen la diferenciación entre dermatitis atópica y xerosis; ${ }^{\star \star \star}$ : no hacen la diferenciación entre onicomicosis y otras micosis.

se reportó una prevalencia de $8,9 \%$ en un grupo de estudio de 214 pacientes con síndrome de Down, con mayor frecuencia en las mujeres que en los hombres $(17,4 \% \text { Vs. } 3,1 \%)^{17}$. No obstante, esta observación no ha sido confirmada por otros autores, como fue el caso de Wunderlich y Braun-Falco, quienes encontraron solo 13 casos (o,13\%) de alopecia areata en 1.000 niños con este síndrome ${ }^{2,25}$. Esta diferencia podría explicarse por los diversos rangos de edad de los pacientes incluidos en cada estudio. La causa no ha sido dilucidada, pero se piensa que por el déficit de linfocitos $\mathrm{T}$ y la disminución de la IgG, se presentan cambios inmunológicos que juegan un papel importante en la etiopatogenia ${ }^{21}$.

Se piensa que esta asociación puede deberse a que la copia adicional del cromosoma 21 podría involucrar genes asociados en la patogénesis. El gen MX1 es el encargado de la síntesis del IFNg inducido por la proteína p78 (MxA), cuya expresión alrededor de las zonas de alopecia está aumentada. En un estudio de 155 pacientes con alopecia areata y 520 controles, se encontró una asociación estadísticamente significativa entre la expresión del gen MX1 y la aparición a más temprana edad de las lesiones ${ }^{21,26}$. La alopecia areata tiende a ser más grave en el síndrome de Down, con presentaciones 
más extensas como la alopecia total y la universal, hasta en 2,5\% de los casos; también, suele ser más resistente al tratamiento estándar. Se ha visto asociación con vitiligo, tiroiditis, hipotiroidismo y traquioniquia, como ocurre en la población sin síndrome de Down ${ }^{14,21}$.

\section{Vitiligo}

El vitiligo es una afección cutánea común que afecta a $1 \%$ de la población en general. Carter y Jegasothy, en su serie, encontraron una prevalencia levemente aumentada, de $1,9 \%{ }^{17}$. El vitiligo puede asociarse con enfermedades autoinmunitarias, lo que explicaría su incidencia ligeramente superior en la población con síndrome de Down ${ }^{14}$.

\section{Queratodermatosis}

En el síndrome de Down, la piel en la infancia es generalmente suave, pero evoluciona rápidamente a una textura gruesa y áspera, con áreas con liquen y xerosis generalizada ${ }^{14}$.

La hiperqueratosis palmo-plantar es una condición en la cual la queratina se engruesa. En los pacientes con síndrome de Down, se inicia después de los cinco meses de edad y tiene una prevalencia de $40 \%$, que aumenta a $75 \%$ después de los cinco años de edad ${ }^{22}$. La xerosis se ha reportado en 9,8\% de los casos, en el rango de edad de dos meses a 25 años, incrementándose su incidencia a $85 \%$ después de los 12 años; además, puede agravarse por la presencia frecuente de hipotiroidismo en estos pacientes (FIGURA 1) 17,22. La asociación del síndrome de Down con ictiosis aún sigue sin ser muy clara; parece que los casos encontrados se deben a coincidencias 0 error en el diagnóstico, al confundirse con xerosis ${ }^{27}$. Los hallazgos de xerosis e hiperqueratosis palmo-plantar se han atribuido a deficiencia de la vitamina A, pero esto no se ha confirmado en estudios posteriores ${ }^{17}$.

La queratosis pilar también tiene una conocida asociación con el síndrome de Down, en el cual su incidencia es de $11,8 \%$ a $33 \%^{2}$, con predominio en el sexo femenino $^{18,20}$. La pitiriasis rubra pilaris y las formas hiperqueratósicas de psoriasis, aunque se han reportado en asociación con este síndrome, parecen tener un curso normal y no hay evidencia concluyente de una asociación mayor ${ }^{21}$, aunque un estudio del 2003 reportó una frecuencia de $5,2 \%{ }^{28}$.

\section{Infecciones cutáneas}

Los pacientes con síndrome de Down son muy propensos a las infecciones cutáneas, debido a las alteraciones ya comentadas del sistema inmunitario, tales como disminución de linfocitos T e IgG. Además, la intensa xerosis presente también juega un rol muy importante en esta predisposición. Es frecuente encontrar infecciones bacterianas a repetición, como foliculitis, forunculosis, abscesos e impétigo secundario. Schepis, et al., reportaron que la foliculitis es la manifestación dermatológica más común². En 1978, Finn, et al., describieron una dermatosis en $45 \%$ de los hombres con síndrome de Down, la cual consistía en pápulas foliculares en zonas seborreicas, la cual describieron como una "dermatosis rara" y la catalogaron como una variante de la dermatitis seborreica, al no encontrar una posible causa ${ }^{23}$.

Posteriormente, Kavanagh et al., en 1993, publicaron un estudio de 22 pacientes hombres y 20 mujeres con un rango de edad comprendido entre los 24 y los 43 años. Diez (45\%) de los 22 hombres, y dos (10\%) de las mujeres, presentaban foliculitis pápulo-pustular, que se localizaba predominantemente en la parte superior de la espalda y el tórax (zona seborreica). El cuadro clínico mejoró con el uso de $100 \mathrm{mg}$ diarios de itraconazol durante 14 días. Aunque solo se aisló Malassezia furfur en cuatro de doce muestras y no se encontró otro microorganismo causal, se considera que estos pacientes presentan frecuentemente foliculitis por $M$. furfur la cual es resistente a los tratamientos, requiriendo altas dosis de itraconazol. Sin embargo, faltan estudios al respecto ${ }^{23,29}$.

Igualmente, son muy frecuentes las infecciones fúngicas, como onicomicosis, tinea pedis y tinea corporis. De los 214 pacientes examinados por Carter y Jegasothy, 145 tenían onicomicosis $(67,8 \%)$, mientras que 164 tenían tinea pedis $(76,6 \%)^{17}$. Schepis, et al., diagnosticaron onicomicosis en $9(4 \%)$ y tinea corporis en 4 ( $2 \%)$ de los 203 pacientes con síndrome de Down ${ }^{2}$. Los autores plantearon la hipótesis de que las infecciones por hongos pueden haber sido asociadas a las condiciones de higiene personal y de la vivienda. El tratamiento para la onicomicosis en estos pacientes no ha sido bien estudiado pues son excluidos de los grupos de estudios; hasta el momento, la terbinafina es la que mejores resultados ha producido ${ }^{30}$.

La escabiosis es otra infección frecuente y los pacientes con síndrome de Down parecen estar más predispuestos a la escabiosis costrosa (sarna noruega) ${ }^{18}$. La sarna noruega es una zooparasitosis de escasa incidencia y muy contagiosa, descrita por primera vez por Danielssen y Boeck en enfermos leprosos de Noruega, en 1848 . Se caracteriza por la proliferación masiva de Sarcoptes scabiei var. hominis, que afecta de forma prevalente a los pacientes con alteraciones inmunitarias y a aquellos con trastornos neurológicos con bloqueo de la sensación de prurito, lo cual lleva a la infestación por 

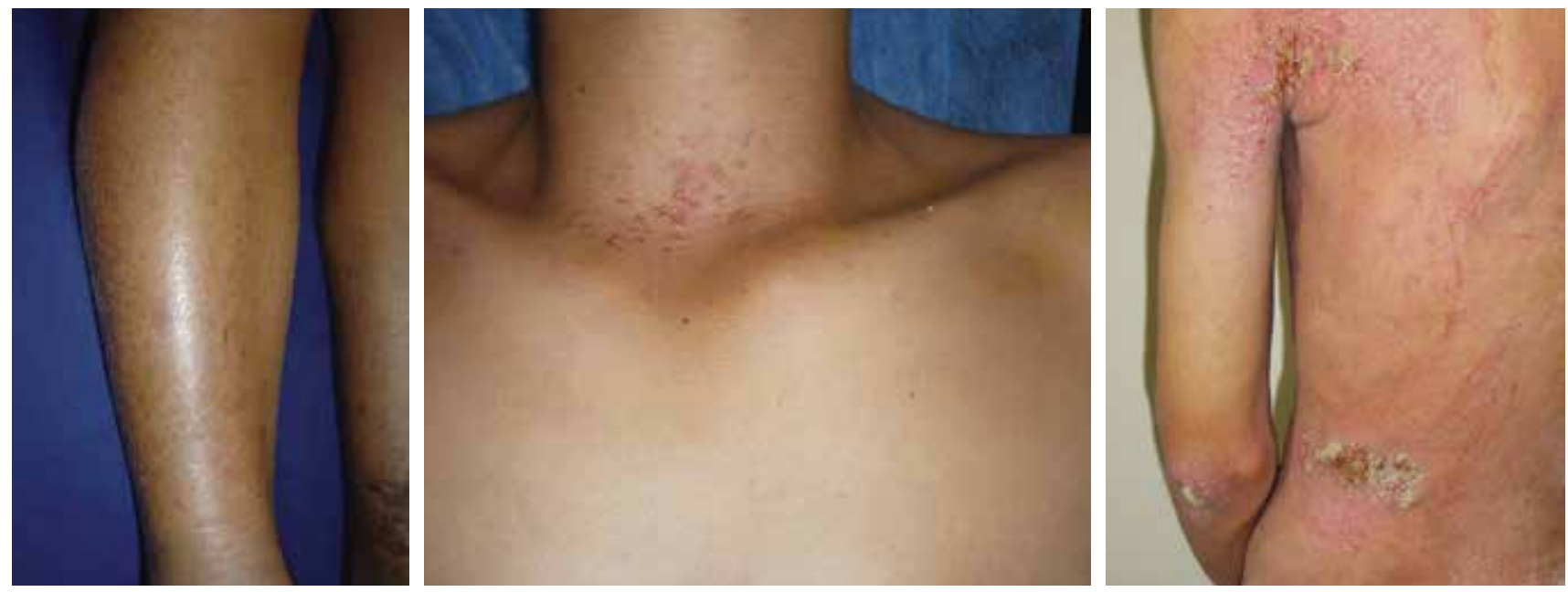

FigURA 1. Las manifestaciones cutáneas en el síndrome de Down incluyen xerosis e ictiosis (A), siringomas eruptivos (B) y sarna noruega (C), entre otras.

el ácaro al disminuir su eliminación mecánica. Estas características las presentan los pacientes infectados por el virus de la inmunodeficiencia humana (VIH), ancianos institucionalizados y personas con síndrome de Down ${ }^{31}$. Las lesiones son típicamente hiperqueratósicas y su localización varía en relación con la forma típica, afectando la cara, el cuero cabelludo y las uñas (FIGURA 1). En casos extremos se ha llegado a describir eritrodermia con eosinofilia y poliadenopatías ${ }^{14}$.

\section{Anetodermia}

También conocida como atrofia macular, consiste en pápulas atróficas que se hernian hacia el interior con la palpación. Histológicamente, se caracteriza por la pérdida del tejido elástico dérmico ${ }^{32}$. En los pacientes con síndrome de Down se ha informado que es secundaria a la foliculitis crónica ${ }^{14,21}$. Existe la hipótesis de que en esta población se presentan malformaciones en la fibras elásticas que pueden ser responsables de la anetodermia ${ }^{21}$. También se ha reportado asociada con alopecia areata, vitiligo e hipotiroidismo, condiciones vistas con mayor frecuencia en el síndrome de Down ${ }^{33}$. El tratamiento opotuno de la foliculitis podría prevenir el desarrollo de anetodermia en esta población.

\section{Alteraciones vasculares}

La acrocianosis y la cutis marmorata ocurren con mayor frecuencia en pacientes con síndrome de Down, princi- palmente en la infancia. La frecuencia de cutis marmorata es de $8 \%$ (edad media de dos años) y la de livedo reticularis es de $11 \% 0^{2,22}$.

La cutis marmorata y la acrocianosis son probablemente más comunes en la infancia en síndrome de Down, a causa de una pobre circulación periférica y aumento en la incidencia de la sífilis congénita y cardiopatías ${ }^{18}$.

\section{Carotenemia}

Es un hallazgo frecuente en pacientes con retardo mental, incluidos aquellos con síndrome de Down. Esto podría explicarse por el hipotiroidismo que con frecuencia se asocia, ya que éste lleva a un aumento de los carotenos en sangre y disminución de su absorción intestinal, lo que posiblemente disminuye la conversión hepática de carotenos a vitamina A. También, se ha postulado que puede estar relacionada con la dieta que estos pacientes reciben en las instituciones ${ }^{18,21,34}$.

\section{Manifestaciones orales}

Los pacientes con síndrome de Down tienen muchas manifestaciones orales distintivas, entre ellas la macroglosia y la lengua geográfica que se presenta hasta en $80 \%$ de los pacientes. La cavidad oral parece ser menor, con prognatismo y mala oclusión dental. La prevalencia de la enfermedad periodontal es alta, y se encuentra lengua fisurada en $28 \%$, hipertrofia papilar de la lengua 
en $22 \%$ y queilitis en $13 \%$ de los casos. Estos pacientes también tienen una mayor tendencia a infección orofacial por cándida ${ }^{35}$.

\section{Dermatosis típicamente relacionadas con el síndrome de Down}

\section{Elastosis perforans serpiginosa}

Las enfermedades perforantes incluyen una variedad de procesos caracterizados por la eliminación transepidérmica. Suelen incluirse en este grupo cinco enfermedades: foliculitis perforans, colagenosis perforans reactiva, elastosis perforans serpiginosa, enfermedad de Kyrle y dermatosis perforans adquirida asociada a diabetes mellitus o insuficiencia renal crónica ${ }^{36}$.

La elastosis perforans serpiginosa es una dermatosis crónica poco frecuente, sin manifestaciones no cutáneas. Es más frecuente en hombres jóvenes, principalmente entre la primera y segunda décadas, y se afirma que $90 \%$ de los pacientes son menores de 30 años $^{37,38}$.

La mayoría de las publicaciones son sobre casos aislados o pequeñas series, lo que refleja mayor riesgo de elastosis perforans serpiginosa en el síndrome de Down, pero no su verdadera frecuencia. En una publicación se reporta una frecuencia de asociación entre el síndrome de Down y la elastosis perforans serpiginosa de $1 \%{ }^{39}$; sin embargo, hay series sin ningún caso de esta enfermedad ${ }^{22}$.

Actualmente, la elastosis perforans serpiginosa se clasifica en tres grupos: la idiopática, la reactiva y la inducida por medicamentos. En la idiopática no hay enfermedad de base; algunos autores afirman que se debe a una predisposición genética. La reactiva corresponde a $25 \%$ de los $\operatorname{casos}^{36}$ y se asocia con alteraciones del tejido conjuntivo, como en el síndrome de Down, el síndrome de Ehlers-Danlos, el síndrome de Marfan, el pseudoxantoma elástico, el síndrome de RothmundThompson, la osteogénesis imperfecta, la esclerosis sistémica y la morfea ${ }^{40}$. La inducida por medicamentos la produce la penicilamina ${ }^{36,37}$.

Las lesiones en la piel se caracterizan por pápulas umbilicadas, eritematosas o de color piel, con una zona central queratósica adherida, que se agrupan formando placas arciformes o serpiginosas, aunque pueden agruparse de forma irregular con lesiones satélites o a distancia. También, se pueden agrupar con un patrón lineal, sugiriendo un fenómeno de Koebner $^{37}$. Estas lesiones suelen estar limitadas ana- tómicamente y raramente son generalizadas. Las localizaciones más frecuentes son la nuca y las zonas laterales del cuello, seguidas de las extremidades superiores y la cara, pero puede haber compromiso de miembros inferiores y tronco. La distribución simétrica es característica, excepto en los casos asociados al síndrome de Down ${ }^{22}$.

La evolución clínica es variable, y las pápulas pueden persistir entre seis meses y cinco años con remisión espontánea, dejando áreas cicatriciales hipopigmentadas, atróficas, con piel arrugada, lineales o reticuladas ${ }^{36}$. Los pacientes con síndrome de Down parecen tener predisposición a desarrollar formas más extensas y crónicas de elastosis perforans serpiginosa con una media del tiempo de evolución de 10 años ${ }^{40}$.

En la histolopatolgía de la elastosis perforans serpiginosa se encuentra aumento focal de las fibras elásticas en la dermis papilar y reticular, más gruesas de lo normal. Estas fibras son eliminadas a través de estrechos canales de perforación transepitelial, transfolicular o parafolicular que contienen un material necrobiótico, basófilo, formado por células epiteliales degeneradas, restos de células inflamatorias y numerosas fibras elásticas, que han perdido sus propiedades de tinción características. El tapón superficial está compuesto de queratina y restos basófilos. Además, ocasionalmente se pueden observar células gigantes a cuerpo extraño en la dermis superficial ${ }^{36}$.

Su patogenia se desconoce. La expulsión a través de la epidermis de fibras elásticas dérmicas, anormales estructural y bioquímicamente, podría ser el resultado de una alteración genética del tejido conjuntivo. Las fibras elásticas alteradas actúan como un material extraño, provocando una reacción tisular que trata de eliminarlo a través de conductos transepidérmicos. Apoya este hecho encontrar depósitos de inmunoglobulinas (IgG, IgA, IgM) y C3 en la dermis papilar. Sin embargo, no hay estudios que confirmen estos hallazgos ${ }^{41}$.

La causa de la relación de la elastosis perforans serpiginosa con el síndrome de Down no está clara, pero junto con otras alteraciones que se observan en este síndrome, como la acrocianosis, la hiperlaxitud articular y el envejecimiento cutáneo prematuro, indicaría una displasia del tejido conjuntivo ${ }^{21}$. La mayor frecuencia de anetodermia secundaria a foliculitis crónica, descrita en el síndrome de Down se ha atribuido a una malformación congénita de las fibras elásticas. Los casos publicados de asociación del síndrome de Down y alteraciones hereditarias del tejido conjuntivo, como el síndrome de Ehlers-Danlos, apoyarían esta hipótesis ${ }^{36}$.

La disfunción inmunitaria de los mecanismos de fagocitosis de estos pacientes, actuaría como un factor favo- 

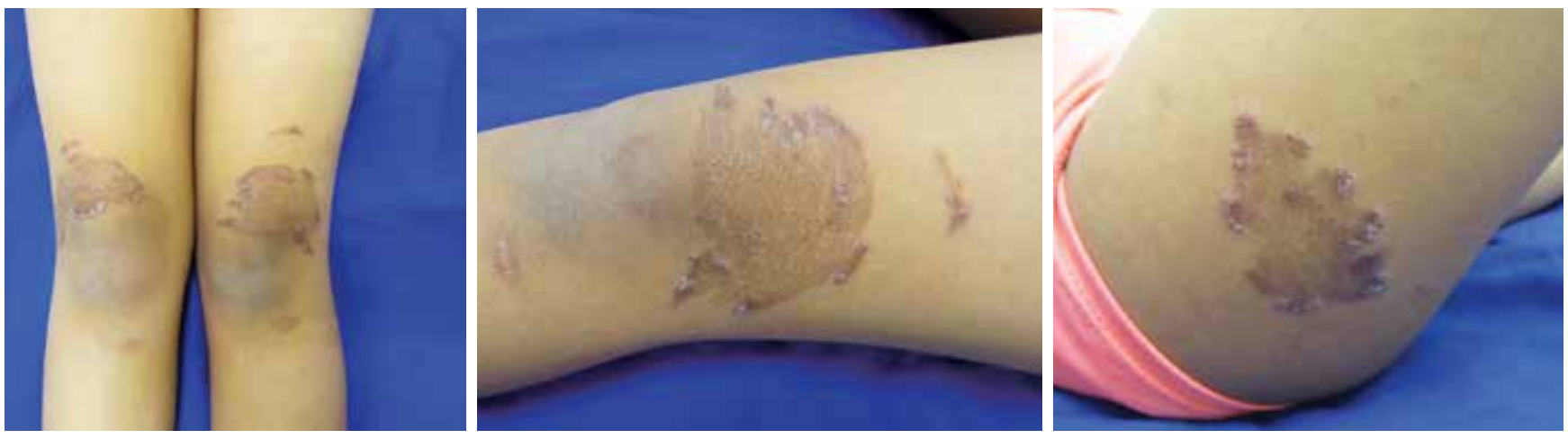

FIGURA 2A. Elastosis perforans serpiginosa en un paciente con síndrome de Down. Placas conformadas por pápulas eritematosasvioláceas que adoptan una configuración anular. Crecimiento centrífugo que deja en el centro la piel adelgazada e hiperpigmentada.
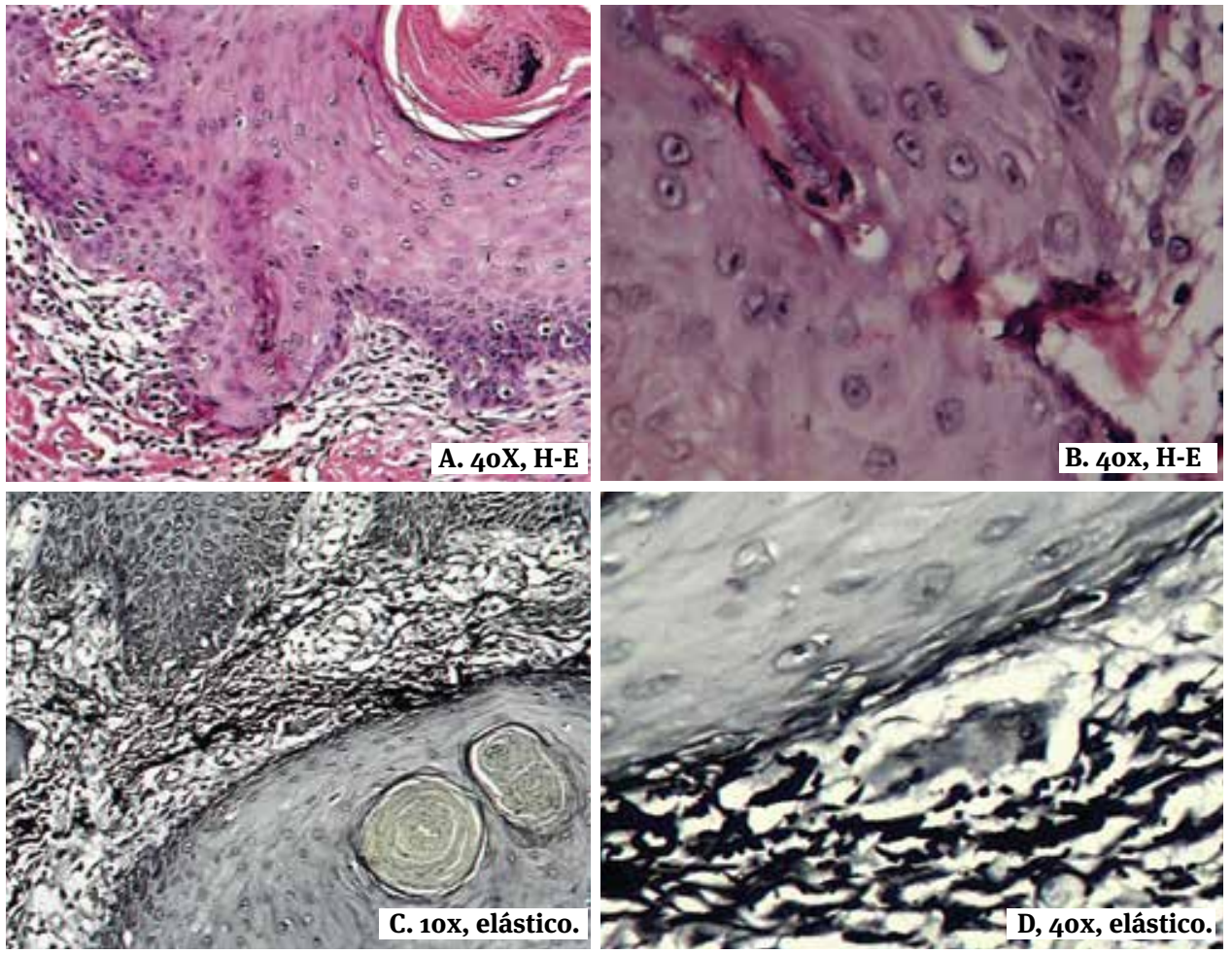

FIGURA 2B. Histopatología de la elastosis perforans serpiginosa.

A. Tapón córneo folicular, con detritos celulares, hiperplasia epitelial folicular con pequeño trayecto de eliminación transepidérmica.

B. Acercamiento del trayecto epitelial.

C. Coloración de elástico donde se aprecian abundantes fibras elásticas gruesas y fragmentadas, con algunas células gigantes multinucleadas de tipo cuerpo extraño.

D. Detalle de las fibras elásticas y de las células gigantes multinucleadas.

recedor del aumento de la eliminación transepidérmica, que representa una vía alternativa para la eliminación de restos celulares. También, se ha descrito un posible agravamiento de las lesiones por déficit de vitamina A en los pacientes con trisomía del cromosoma 21 y elastosis perforans serpiginosa ${ }^{41}$.

Para la elastosis perforans serpiginosa se han utilizado múltiples tratamientos con resultados variables y ninguno es universalmente aceptado. Es difícil su evaluación por su escasa frecuencia y la posibilidad de remisión espontánea.

Se han usado tratamientos tópicos con corticoides, queratolíticos, retinoides como el tazaroteno al o,1\%, imiquimod en crema y calcipotrol, tratamientos sistémicos con isotretinoina oral y diversas técnicas quirúrgicas (electrocoagulación, crioterapia o láser) ${ }^{36,37}$.

En la Figura 2 se ilustra el caso de una paciente de ocho años de edad con elastosis perforans serpiginosa asociada a síndrome de Down, con antecedentes de hipogammaglobulinemia, hipotiroidismo subclínico, cirugía de corrección de conducto arterioso persistente y de estenosis de aorta, miringotomía y amigdalectomía. Recibió tratamientos con metoprolol, espirinolactona y beclometasona en inhalador nasal. 
Fue producto del segundo embarazo y nació por cesárea a las 36 semanas de gestación por preeclampsia. No tenía antecedentes familiares de importancia. Consultó por lesiones de cuatro años de evolución que se iniciaron en las rodillas como placas que crecían centrífugamente, se extendieron a los muslos y se asociaron a leve prurito.

En el examen físico se encontró en buenas condiciones generales, con placas conformadas por pápulas eritematosas que adoptaban una configuración anular, con crecimiento centrífugo, adelgazamiento de la piel en su centro e hiperpigmentadas. La biopsia de piel confirmó el diagnóstico al observarse eliminación transepidérmica de las fibras elásticas.

Recibió tratamiento tópico con esteroides, tazaroteno, humectantes con úrea y calcipotriol, con escasa mejoría, por lo que se inició isotretinoína oral con lo cual ha disminuido la progresión de las placas y no han aparecido lesiones nuevas.

\section{Calcinosis cutis de tipo miliar}

Es un subtipo de calcinosis cutánea idiopática, descrito por primera vez en 1978. En una revisión del 2007 de los casos reportados, se encontraron 19 pacientes con calcinosis cutis tipo miliar, 6 de los cuales se asociaban con síndrome de Down, con una edad promedio de 10 años ${ }^{42}$.

Las lesiones aparecen como pápulas discretas, blancas o de color piel, pero firmes y con aspecto calcáreo, algunas rodeadas por eritema; y también se pueden encontrar algunas con una costra central que corresponden a eliminación transepidérmica de calcio $^{43}$. Los pacientes son asintomáticos y las manos y los pies se afectan más comúnmente, pero puede aparecer en cualquier parte del cuerpo ${ }^{21}$. No se encuentran alteraciones del metabolismo del calcio ni anormalidades anteriores a la lesión tisular, pero pueden asociarse a siringomas palpebrales o perilesionales ${ }^{43}$. En la histopatología se observan nódulos basófilos en la dermis papilar, que corresponden a depósitos de calcio redondos y bien definidos que pueden estar rodeados por fibras de colágeno gruesas, células epiteliales y células gigantes multinucleares ${ }^{43}$.

Aun no es clara la patogenia de la asociación de esta enfermedad con el síndrome de Down. Se habla de mayores concentraciones de calcio en el sudor, que llevan a la calcificación del conducto sudoríparo; también, se ha encontrado mayor concentración de calcio en los cultivos de fibroblastos de pacientes con síndrome de Down. El aumento del calcio puede ser un factor que predispone al desarrollo de las lesiones dérmicas. Otra hipótesis propone que las lesiones representan microquistes epidérmicos, que posteriormente generan un reacción inflamatoria crónica con depósitos de calcio ${ }^{44}$.

Estas lesiones suelen mejorar y curar con el tiempo, con cicatriz o sin ella, antes de la adultez, por lo que no se recomienda ningún tratamiento ${ }^{21}$.

\section{Siringomas}

Los siringomas son neoplasias benignas con diferenciación ductal acrosiríngea, que se presentan como pápulas firmes, de color piel normal o amarillenta, de tamaño variable, entre 1 y $5 \mathrm{~mm}$, aunque la mayoría son de menos de $3 \mathrm{~mm}$. Generalmente, son múltiples y con distribución bilateral y simétrica (FIGURA 1 ). Pueden aparecer en cualquier parte del cuerpo, pero tienen predilección por la región periorbitaria, en especial por los párpados inferiores y la región malar. En ocasiones se presentan en el tronco y en el área genital. En la histopatología se observan tumores dérmicos sin conexión con la epidermis, compuestos por: a) múltiples conductos pequeños tapizados por dos capas de epitelio cúbico, que ocasionalmente pueden estar dilatados y contener material eosinófilo, o bien, adquirir forma de coma que recuerda al tricoepitelioma desmoplásico; b) nidos sólidos y cordones de células epiteliales de aspecto basaloide, y c) estroma denso fibroso, de aspecto escleroso ${ }^{45}$.

Se sabe que la incidencia de siringomas en el síndrome de Down es mucho mayor que en la población general; en diferentes estudios se han encontrado incidencias entre 6 y $30 \%$, con una relación mujer a hombre de 2:145,46. También, se ha notado la asociación de siringomas con calcinosis cutis idiopática, lo que apoya la hipótesis de que la estructura del conducto intraepidérmico puede desempeñar un papel importante en la patogénesis de la calcinosis idiopática de tipo miliar en el síndrome de Down.

Las opciones terapéuticas incluyen electrocoagulación o crioterapia. El ácido retinoico tópico y la atropina se han utilizado para tratar siringomas eruptivos ${ }^{45}$.

\section{Leucemia cutis}

Los recién nacidos con trisomía 21 tienen un mayor riesgo de alteraciones hematológicas benignas, incluyendo trastornos transitorios mieloproliferativos y reacción leucemoide; también, están en mayor riesgo de leucemia congénita ${ }^{47,48}$. El riesgo de leucemia en el síndrome de Down es de 1 a $1,5 \% 49$.

La leucemia cutis se asocia a menudo con problemas congénitos de leucemia, principalmente con leucemia mieloide aguda ${ }^{48,50}$. En cuanto a las manifestaciones clínicas, se pueden presentar en cualquier parte del cuerpo, con menor frecuencia aparecen en zonas 


\section{Caracteristicas clinicas de paciente con trisomia 21}

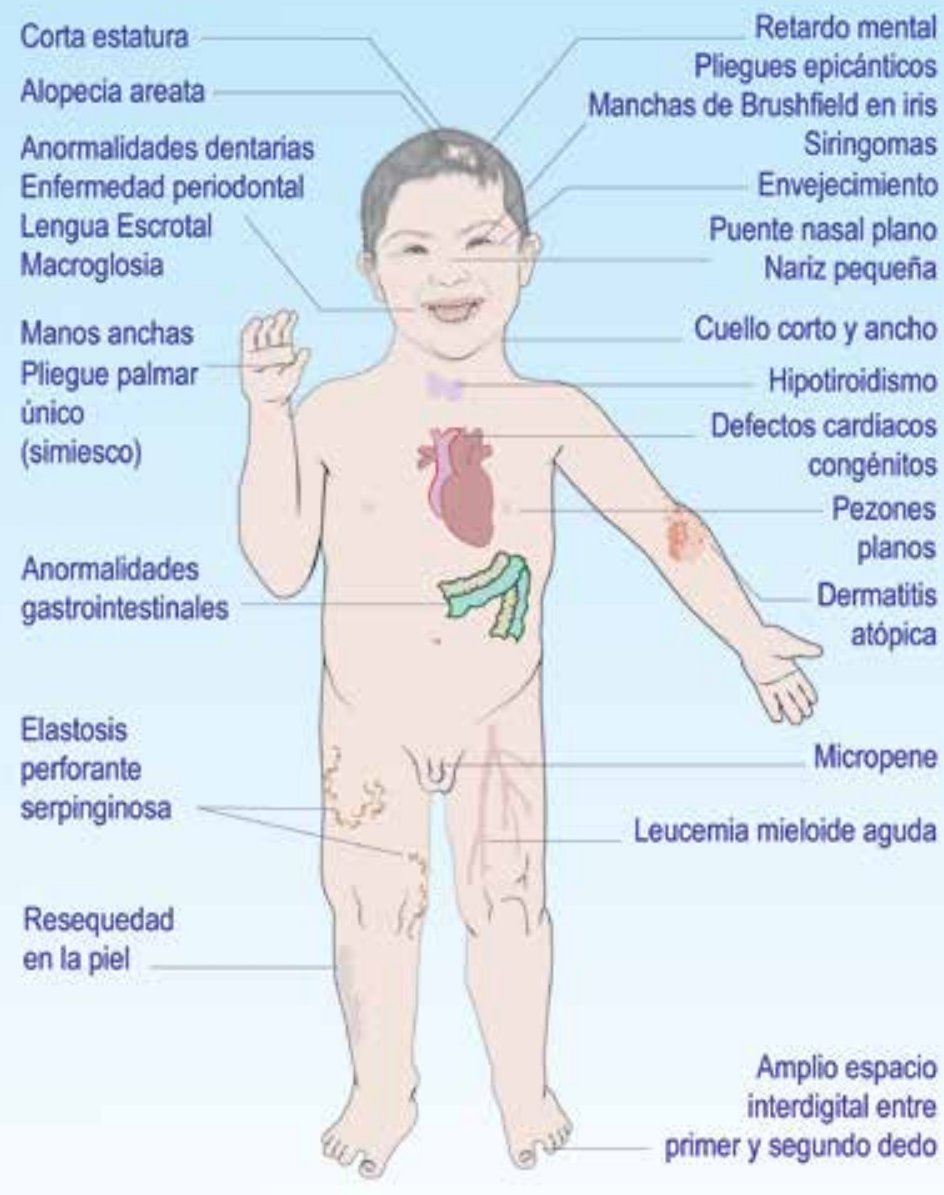

FIGURA 3. Manifestaciones cutáneas del síndrome de Down. Ilustración adaptada con permiso del autor. Modificada de: Cammarata F, Piquero-Casals J. Alteraciones dermatológicas en el síndrome de Down. Dermatología Venezolana 2005; $43: 4-6$ palmo-plantares y en la mucosa oral. Las lesiones clínicas pueden ser solitarias o múltiples, circunscritas o diseminadas; esta última distribución puede ser un indicio de una forma aguda de leucemia. Los hallazgos clínicos más comunes en la leucemia cutis son pápulas, placas, nódulos o lesiones tumorales. Los grandes nódulos pueden llegar a medir varios centímetros y tener consistencia cauchosa. A veces, los tumores pueden ser tan grandes como un puño y también pueden estar cubiertos con costras o escamas, con o sin ulceración. Las lesiones varían de color desde amarillo a marrón, rojo o púrpura $^{50}$. Se han descrito erupciones inusuales, como pústulas o lesiones vesiculo-pustulosas, en niños con síndrome de Down en asociación con la reacción leucémica y alteraciones mieloproliferativas ${ }^{47,48}$.

Desde el punto de vista histopatológico, la leucemia cutis se caracteriza por infiltrado dérmico perivascular e infiltrado intersticial de las células leucémicas que puedan extenderse profundamente en el tejido subcutáneo. Es posible que haya infiltración y destrucción de anexos ${ }^{50}$.

En la FIGURA 3 se ilustran las manifestaciones cutáneas del síndrome de Down.

\section{Conclusión}

Las manifestaciones dermatológicas en el síndrome de Down son múltiples y le corresponde al especialista identificarlas y tratarlas. Los cuidados de la piel del paciente con esta condición deben incluir una adecuada lubricación y aseo para evitar procesos infecciosos secundarios. Las características fenotípicas propias de la 
piel y mucosas en estos pacientes, deben explicarse a los familiares para evitar angustia y confusión.

El trabajo médico multidisciplinario preventivo es fundamental para el desarrollo saludable del individuo con dicha condición y el dermatólogo debe constituirse en pieza fundamental para mantener la piel, las mucosas y los anexos en condiciones óptimas y sin enfermedad.

\section{Referencias}

1. Bull MJ. Health supervision for children with Down syndrome. Pediatrics. 2011;128:393-406.

2. Schepis C, Barone C, Siragusa M, Pettinato R, Romano C. An updated survey on skin conditions in Down syndrome. Dermatology (Basel, Switzerland). 2002;205:234-8.

3. Garrison MM, Jeffries H, Christakis DA. Risk of death for children with down syndrome and sepsis. J Pediatr. 2005;147:748-52.

4. Cruz NV, Mahmoud SA, Chen H, Lowery-Nordberg M, Berlin K, Bahna SL. Follow-up study of immune defects in patients with dysmorphic disorders. Ann Allergy Asthma Immunol. 2009;102:426-31.

5. Kusters M, Verstegen RHJ, Gemen EF, de Vries E. Intrinsic defect of the immune system in children with Down syndrome: A review. Clin Exp Immunol. 2009;156:189-93.

6. de Hingh YCM, van der Vossen PW, Gemen EFA, Mulder AB, Hop WCJ, Brus F, et al. Intrinsic abnormalities of lymphocyte counts in children with down syndrome. J Pediatr. 2005;147:744-7.

7. Nisihara RM, Utiyama SRR, Oliveira NP, Messias-Reason IJ. Mannan-binding lectin deficiency increases the risk of recurrent infections in children with Down's syndrome. Hum Immunol. 2010;71:63-6.

8. Yasui K, Shinozaki K, Nakazawa T, Agematsu K, Komiyama A. Presenility of granulocytes in Down syndrome individuals. Am J Med Genet. 1999;84:406-12.

9. Ram G, Chinen J. Infecciones e inmunodeficiencia en el síndrome de Down. Revista Síndrome de Down. 2011;28:70-81.

10. Larocca LM, Piantelli M, Valitutti S, Castellino F, Maggiano N, Musiani P. Alterations in thymocyte subpopulations in Down's syndrome (trisomy 21). Clin Immunol Immunopathol. 1988;49:175-86.

11. Cocchi G, Mastrocola M, Capelli M, Bastelli A, Vitali F, Corvaglia L. Immunological patterns in young children with Down syndrome: Is there a temporal trend? Acta Paediatr. 2007;96:1479-82.

12. Roat E, Prada N, Lugli E, Nasi M, Ferraresi R, Troiano L, et al. Homeostatic cytokines and expansion of regulatory $\mathrm{T}$ cells accompany thymic impairment in children with Down syndrome. Rejuvenation Res. 2008;11:573-83.

13. Zerhouni E. Health conditions associated with aging and end of life of adults with Down syndrome. Science. 2004;39:1-15.

14. Barankin B, Guenther L. Dermatological manifestations of Down's syndrome. J Cutan Med Surg. 2001;5:289-93.

15. Hart RW, Setlow RB. Correlation between deoxyribonucleic acid excision-repair and life-span in a number of mammalian species. Proc Natl Acad Sci USA. 1974;71:2169-73.

16. Sinha S. Anti-oxidant gene expression imbalance, aging and Down syndrome. Life Sci. 2005;76:1407-26.

17. Carter DM, Jegasothy BV. Alopecia areata and Down syndrome.
Arch Dermatol. 1976;112:1397-9.

18. Scherbenske JM, Benson PM, Rotchford JP, James WD. Cutaneous and ocular manifestations of Down syndrome. J Am Acad Dermatol. 1990;22:933-8.

19. Schepis C, Barone C, Siragusa M, Romano C. Prevalence of atopic dermatitis in patients with Down syndrome: A clinical survey. J Am Acad Dermatol. 1997;36:1019-21.

20. Cano MP. La piel y su expresión en la clínica del niño con sín drome de Down. Rev Med Int Sindr Down. 2011;15:23-5.

21. Madan V, Williams J, Lear JT. Dermatological manifestations of Down’s syndrome. Clin Exp Dermatol. 2006;31:623-9.

22. Ercis M, Balci S, Atakan N. Dermatological manifestations of 71 Down syndrome children admitted to a clinical genetics unit. Clinical Genetics. 1996;50:317-20.

23. Kavanagh GM, Leeming JP, Marshman GM, Reynolds NJ, Burton JL Folliculitis in Down's syndrome. Br J Dermatol. 1993;129:696-9.

24. Alkhalifah A, Alsantali A, Wang E, McElwee KJ, Shapiro J. Alopecia areata update: Part I. Clinical picture, histopathology, and pathogenesis. J Am Acad Dermatol. 2010;62:177-188.

25. Wunderlich C, Braun-Falco O. Mongolism and alopecia areata. Med Welt. 1965;10:477-81

26. Tazi-Ahnini R, di Giovine FS, McDonagh AJ, Messenger AG, Amadou C, Cox A, et al. Structure and polymorphism of the human gene for the interferon-induced p78 protein (MX1): Evidence of association with alopecia areata in the Down syndrome region. Hum Genet. 2000;106:639-45.

27. Kopec AV, Levine N. Generalized connective tissue nevi and ichthyosis in Down's syndrome. Arch Dermatol. 1979;115:623-4.

28. Ferrando J, Escobar CM. Incidencia de patología dermatológica en los pacientes del Centre Mèdic Down de la Fundació Catalana Síndrome de Down. Revista Médica Internacional sobre el Síndrome de Down. 2003;7:39-43.

29. Finn OA, Grant PW, McCallum DI, Raffle EJ. A singular dermatosis of Mongols. Arch Dermatol. 1978;114:1493-4.

30. Barber K, Claveau J, Thomas R. Review of treatment for onychomycosis: Consideration for special populations. J Cutan Med Surg. 2006;10(Suppl.2):S48-53.

31. Ruiz Villaverde R, Blasco Melguizo J, Fernández Pugnaire MA, Allegue Gallego F, Delgado Florencio V. Lesiones hiperqueratósicas en paciente con síndrome de Down. Rev Clin Esp. 2002;202:6156.

32. Lewis KG, Bercovitch L, Dill SW, Robinson-Bostom L. Acquired disorders of elastic tissue: Part II. Decreased elastic tissue. J Am Acad Dermatol. 2004;51:165-85.

33. Hodak E, Shamai-Lubovitz O, David M, Hazaz B, KatzenelsonWeissman V, Lahav M, et al. Immunologic abnormalities associated with primary anetoderma. Arch Dermatol. 1992;128:799803.

34. Pozo Cano MD, González Jiménez E, Álvarez Ferre J, Martínez García E, Navarro Jiménez MC. La piel y su expresión en la clínica del niño con síndrome de Down. Rev Med Int Sindr Down. 2011;15:23-5

35. Scully C, van Bruggen W, Diz Dios P, Casal B, Porter S, Davison M-F. Down syndrome: Lip lesions (angular stomatitis and fissures) and Candida albicans. Br J Dermatol. 2002;147:37-40.

36. Pereira AC, Baeta IG, Costa Júnior SR, Gontijo Júnior OM, Vale EC.Elastosis perforans serpiginosa in a patient with Down's. An Bras Dermatol. 2010 Sep-Oct;85(5):691-4. 
37. Arredondo MI, Londoño A, Restrepo R. Enfermedades con eliminación transepidérmica. Rev Asoc Col Dermatol. 2008;16:185-95.

38. Yáñez S, Val-Bernal JF, Riancho JA. Elastosis perforante serpiginosa en pacientes con síndrome de Down. Med Cutan Iber Lat Am. 2010;38:234-7.

39. Rasmussen JE. Disseminated elastosis perforans serpiginosa in four mongoloids. Recognition of residual changes. Br J Dermatol. 1972;86:9-13.

40. Mehta RK, Burrows NP, Payne CM, Mendelsohn SS, Pope FM, Rytina E. Elastosis perforans serpiginosa and associated disorders. Clin Exp Dermatol. 2001;26:521-4.

41. Valdivielso M, Lecona M, Suárez R. Elastosis perforante serpiginosa de localización inusual en un paciente con síndrome de Down. Actas Dermosifiliogr 2004;95:171-4.

42. Houtappel M, Leguit R, Sigurdsson V. Milia-like idiopathic calcinosis cutis in an adult without Down's syndrome. J Dermatol Case Rep. 2007;1:16-9.

43. Jang EJ, Lee JY, Yoon TY. Milia-like idiopathic calcinosis cutis occurring in a toddler born as a premature baby. Ann Dermatol. 2004;23:490-2.

44. Kim DH, Kang H, Cho SH, Park YM. Solitary milialike idiopathic calcinosis cutis unassociated with Down's syndrome: Two case reports. Acta Derm Venereol. 2000;80:151-2.

45. Ong GCW, Lim KS, Chian LYT. Eruptive syringoma in a patient with trisomy 21. Singapore Med. 2010;51:46-7.
46. Daneshpazhooh M, Nazemi TM, Bigdeloo L, Yoosefi M. Mucocutaneous findings in 100 children with Down syndrome. Pediatr Dermatol. 2007;24:317-9.

47. Solky BA, Yang FC, Xu X, Levins P. Transient myeloproliferative disorder causing a vesiculopustular eruption in a phenotypically normal neonate. Pediatr Dermatol. 2004;21:551-4.

48. Nijhawan A, Baselga E, Gonzalez-Ensenat MA, Vicente A, Southern JF, Camitta BM, et al. Vesiculopustular eruptions in Down syndrome neonates with myeloproliferative disorders. Arch Dermatol. 2001;137:760-3.

49. Malinge S, Izraeli S, Crispino JD. Insights into the manifestations, outcomes, and mechanisms of leukemogenesis in Down syndrome. Blood. 2009;113:2619-28.

50. Wagner G, Fenchel K, Back W, Schulz A, Sachse MM. Leukemia cutis -epidemiology, clinical presentation, and differential diagnoses. J Dtsch Dermatol Ges. 2012;10:27-36.

51. Ferrando J. Caso clínico. Incidencia de patología dermatológica en los pacientes del Centre Mèdic Down de la Fundació Catalana Síndrome de Down. Revista Médica Internacional sobre el Síndrome de Down. 2003;7:39-43.

52. Polenghi MM, Piattoni F, Orsini GB. Dermatologic disorders in Down syndrome. Am J Med Genet Suppl. 1990;7:324.

53. Martin D. Alopecia areata and Down syndrome. Arch Dermatol. 1976;7:19-21. 